\title{
Reviewer Acknowledgments
}

\section{Principal Reviewers}

The Editor and Associate Editors wish to thank the following persons who served as principal reviewers for Psychology of Addictive Behaviors by reviewing a minimum of four manuscripts.

Patrick Bertotti Metoyer

Julia D. Buckner

MaryAnn Chapman

Caitlin Abar

Sue Adams

Joseph L. Alcorn III

Josefina Alvarez

Michael Amlung

Brian A. Anderson

Kristen G. Anderson

Judy A. Andrews

Paul A. Arbisi

Stephen Armeli

Brooke Arterberry

James Ashenhurst

Anu Asnaani

Elizabeth Aston

Steven Babbin

Kimberly A. Babson

Rachel Bachrach

John S. Baer

Jennifer Bailey

Kimberly Balsam

Anne N. Banducci

Anat Bardi

Bruce D. Bartholow

Brian R. Baucom

Jean Beckham

Greg Belenky

Erin C. Berenz

L. Cinnamon Bidwell

Erika Litvin Bloom

Arthur W. Blume

Matthew T. Boden

Krysten Bold

Kaitlin Bountress

Elizabeth Brondolo

Cati Brown-Johnson

Mason Burley

John Cacciola

Christy Capone

Daniel Capron

Beatriz Carlini

Matthew Carpenter

Pilar Carrera

Rachel Cassidy

Naomi Chaytor

Kelvin Choi

Adrienne Heinz

Robert F. Leeman

Molly Magill

\section{Contributing Reviewers}

Paul Christiansen

Joy Chudzynski

Luke Clark

Donna Coffman

Lee M. Cohen

R. Lorraine Collins

Kevin Connolly

Ned L. Cooney

Theodore Cooper

Amy L. Copeland

William R. Corbin

Elizabeth Corby

Jesse Cougle

James A. Cranford

Kasey Creswell

Jessica M. Cronce

Michael Cucciare

John Cunningham

John J. Curtin

Brenda L. Curtis

Melissa A. Cyders

Gayle A. Dakof

Elizabeth J. D'Amico

Jason Daniel-Ulloa

Stacey Daughters

Kelly Cue Davis

Anne M. Day

Michael E. DeBakey

Eric Dedert

Paul Delfabbro

Kelly S. DeMartini

Karen Derefinko

Jeffrey L. Derevensky

Sarah Dermody

Jaye L. Derrick

Arielle Deutsch

Angelo M. DiBello

Lisa Dierker

Karen Dugosh

Laura Dunlap

Kelly E. Dunn

Robert D. Dvorak

Dennis Dyck

Rina Eiden

Jennifer C. Elliott
Jean-François Etter

Elizabeth Evans

Suzette Evans

Tera Fazzino

Stuart G. Ferguson

David Festinger

Brandi Fink

Daniel Fischer

Sarah Fischer

Ben Fitzpatrick

Dawn Foster

Tracey Garcia

Jacques Gaume

Ashley N. Gearhardt

Christian Geiser

Philip Gendall

Meg Gerrard

Peter R. Giancola

Frederick (Rick) X. Gibbons

Bryan Gibson

Christine Gidycz

Joseph Glass

Susan H. Godley

Mark Godley

Jeremy T. Goldbach

Vivian M. Gonzalez

Raul Gonzalez

Adam S. Goodie

Sean Grant

Heather Gray

Mark K. Greenwald

Daniel F. Gros

Casey Guillot

Kevin A. Hallgren

Dennis Hand

Paul Truman Harrell

Larry Hawk

Eric Hawkins

Sandy Henley

James (Matt) M. Henson

Reid K. Hester

Robert Hester

Brian Hicks

Stephen T. Higgins
Jennifer P. Read

Jeremiah Weinstock

Marian Wilson
Chandni Hindocha

Julianna Hogan

Diana S. Hoover

Hyman Hops

Jon M. Houck

Rebecca J. Houston

John Hustad

Jessica Irons

Isabel Jacobson

Lois James

Emily Jeffries

Jennifer M. Jester

Patrick Johnson

Antonia Kaczkurkin

Carl Kantner

Sarah Karalunas

Janet Katz

David Kerr

Serena M. King

Daniel King

Valerie Knopik

John Kramer

Magda Kulesza

Caroline Kuo

Paul Kwon

Benjamin O. Ladd

Cynthia Lakon

Cho Lam

Emilio Landolfi

Scott Lane

Kirsten Langdon

Debi LaPlante

Sherry Larkins

Anne-Marie Laslett

Cathy Lau-Barraco

Matt Layton

Mattthew R. Lee

Chien-Ti Lee

Ellen Leen-Feldner

Carl W. Lejuez

Kathryn Lenberg

Kenneth E. Leonard

Ash Levitt 
Krista M. Lisdahl

Marianne Littel

Jennifer Livingston

Elizabeth Lloyd-Richardson

Daniela Lobo

Mallory J. Loflin

Travis Lovejoy

Jeremy Luk

Jane Luterek

Kevin G. Lynch

Alexandra Macdonald

Kristen Mackiewicz Seghete

James MacKillop

Vance V. MacLaren

Michael G. MacLean

Laura MacPherson

Jennifer L. Maggs

Renee Magnan

Kerry Makin-Byrd

Kimberly Mallett

Cecile A. Marczinski

Katherine Marks

Matthew P. Martens

Rosemarie A. Martin

Ryan J. Martin

Robin Masheb

W. Alex Mason

Nadine R. Mastroleo

Olivia Maynard

Carolyn McCarty

F. Joseph McClernon

Erin A. McClure

Meghan E. McDevitt-Murphy

Matthew McGue

Steve Meredith

Robin J. Mermelstein

Mary Beth Miller

Katherine Mills

Robert Miranda

Brooke S. G. Molina

Kevin S. Montes

Frederick J. Muench

Eun-Young Mun

Marlon P. Mundt
Cara M. Murphy

Bonnie Nagel

Lucy E. Napper

Chantal Nederkoorn

Sonya Norman

Lindsay Margaret-Sander Oberleitner

Sabrina Oesterle

Christine Ohannessian

Lindsay Orchowski

Joseph J. Palamar

Raina Pang

Roger Papke

Julie A. Patock-Peckham

Sarah L. Pedersen

Eric R. Pedersen

Erica Peters

James G. Phillips

Kristina T. Phillips

Geoffrey Potts

Mathew S. Powers

Mark A. Prince

Judith J. Prochaska

Brian M. Quigley

Rajeev Ramchand

Jason J. Ramirez

Jeffery Ramirez

Jorge Ramirez Garcia

Susan Ramsey

Anne E. Ray

Lara Ray

Madhavi Reddy

Florian Rehbein

Alan Reifman

H. Jonathon Rendina

Soo Rhee

John Roache

Maria Roditis

Gail Rose

Craig Rosen
M. Zachary Rosenthal

Steve Rouse

Amy Rubin

Craig Roy Rush

Christopher Salas-Wright

Brendan Saloner

Julie Salvador

Karen K. Saules

Andrew Saxon

Steven Sayers

Michael A. Sayette

Joseph Schacht

Sarah Schmiege

Robert Schnoll

Pamela Schuetze

Lelslie Schuh

Jeremiah Schumm

Katreena Scott

Kennon M. Sheldon

Kenneth J. Sher

Mariya Shiyko

Steve Shoptaw

Raluca M. Simons

Tracy L. Simpson

Anne Helene Skinstad

Andrew Smirnov

Philip H. Smith

Sarah Snider

Gerald Stahler

Jerod L. Stapleton

Cynthia Stappenbeck

Paul R. Stasiewicz

Lynda Stein

Robert S. Stephens

Joseph Studer

Meenakshi Sabina Subbaraman

Tami Sullivan

Marc Swogger

Amelia Talley

Ellen Teng

Linda Thompson

Christine Timko

Rachel Tomko
Sarah L. Tragesser

Hayley R. Treloar

Elisa Trucco

Joan S. Tucker

Sarah E. Ullman

Hans van der Baan

Tony van Rooij

Guido Van Wingen

Roxanne Vandermause

Michael Vaughn

Jennifer Celene Veilleux

Terril Verplaetse

Francesco Versace

Damon J. Vidrine

Olivia Wackowski

Mary Waldron

Kimberly Walitzer

Jeffrey Wardell

Jessica Weafer

Jeff Weatherly

Christian Webb

Nicole Weiss

James P. Whelan

Marney White

Aaron White

John White

Reinout W. Wiers

Claire E. Wilcox

Sam Wilkinson

Robert Williams

Carolyn Wong

Elizabeth Yeater

Kelly Young-Wolff

Martin Zack

James Zacny

Kristyn Zajac

Michael Zvolensky

\section{Contributing Pre-Doctoral Co-Reviewers}

Sarah Bilsky
Brian Calhoun
Nicole Cohen
Martin De Vita
Joshua Gottlieb
Melissa Graham
ReJoyce Green

Katherine Hirchak

Matthew Keough

Salpi Kevorkian

Jueun Kim

Daniella Klebaner

Heather Krieger

Kathryn Lachance

Jennifer Lai

Crystal Lederhos
Connor McCabe

Anas Mohammad

MacKenzie Peltier

Elizabeth Reese

Michael Richards

Nichole Sell

Hannah Shulman

Kayla Skinner
Alicia Starkey

Chelsea Tobin

Bradley Trager

Sonya Wanklyn

Eric Woodcock

Michelle Zaso 